\title{
The AiiDA-Spirit Plugin for Automated Spin-Dynamics Simulations and Multi-Scale Modeling Based on First-Principles Calculations
}

\begin{abstract}
Philipp Rüßmann ${ }^{1 *}$, Jordi Ribas Sobreviela ${ }^{1,2}$, Moritz Sallermann ${ }^{1,2,3}$, Markus Hoffmann ${ }^{1,2}$, Florian Rhiem ${ }^{4}$ and Stefan Blügel ${ }^{1}$

${ }^{1}$ Peter Grünberg Institut (PGI-1) and Institute for Advanced Simulation (IAS-1), Forschungszentrum Jülich, Jülich, Germany, ${ }^{2}$ RWTH Aachen University, Aachen, Germany, ${ }^{3}$ Science Institute and Faculty of Physical Sciences, University of Iceland, VR-III, Reykjavik, Iceland, ${ }^{4}$ Peter Grünberg Institut/Jülich Centre for Neutron Science-Technical Services and Administration (PGI/ JCNS-TA), Forschungszentrum Jülich, Jülich, Germany
\end{abstract}

Landau-Lifshitz-Gilbert (LLG) spin-dynamics calculations based on the extended Heisenberg Hamiltonian is an important tool in computational materials science involving magnetic materials. LLG simulations allow to bridge the gap from expensive quantum mechanical calculations with small unit cells to large supercells where the collective behavior of millions of spins can be studied. In this work we present the AiiDA-Spirit plugin that connects the spin-dynamics code Spirit to the AiiDA framework. AiiDA provides a Python interface that facilitates performing highthroughput calculations while automatically augmenting the calculations with metadata describing the data provenance between calculations in a directed acyclic graph. The AiiDA-Spirit interface thus provides an easy way for high-throughput spin-dynamics calculations. The interface to the AiiDA infrastructure furthermore has the advantage that input parameters for the extended Heisenberg model can be extracted from highthroughput first-principles calculations including a proper treatment of the data provenance that ensures reproducibility of the calculation results in accordance to the FAIR principles. We describe the layout of the AiiDA-Spirit plugin and demonstrate its capabilities using selected examples for LLG spin-dynamics and Monte Carlo calculations. Furthermore, the integration with first-principles calculations through AiiDA is demonstrated at the example of $\gamma-\mathrm{Fe}$, where the complex spin-spiral ground state is investigated.

Keywords: spin-dynamics simulation, high-throughput computation, Landau-Lifshitz-Gilbert equation, Monte-Carlo simulation, spin-spiral state, gamma-Fe, skyrmion, antiskyrmion

\section{INTRODUCTION}

Magnetic materials play an important role in modern technology. Their most important applications range from electrical motors to the storing and processing of digital information. The performance of such applications crucially relies on the performance of magnets where the knowledge of their magnetic order, the Curie temperature, the magnetic hardness or their chirality plays an important role. Computational materials design of magnetic materials and devices is a complex multi-scale 
problem. While quantum mechanical calculations allow to predict the interaction strength among magnetic atoms (Liechtenstein et al., 1987), large scale simulations for nanometer to micrometer length scales are unfeasible due to their computational cost. Mapping these interactions to a classical Heisenberg model allows to bridge the scales from the atomic length scale to the length scale of devices. The classical Heisenberg model is an approximation to the quantum mechanical problem which assumes that the magnetic moments are localized on atoms and can be described as classical vectors which is applicable for a wide range of materials.

Spin-dynamics calculations based on the Landau-LifshitzGilbert (LLG) equation (Landau and Lifshitz, 1935; Gilbert, 2004) are a widely used tool for this multi-scale modeling of magnetic materials (Dupé et al., 2014; Hoffmann et al., 2017; Hoffmann et al., 2021; Weißenhofer et al., 2021), providing access to the collective behavior of millions of spins (Müller et al., 2019). This approach allows to find, for instance, the (non-collinear) magnetic ground state based on an energy minimization of the extended Heisenberg Hamiltonian or to study the dynamics of magnetic solitons such as skyrmions (Muihlbauer et al., 2009; Yu et al., 2010; Heinze et al., 2011; Back et al., 2020) or hopfions (Bogolubsky, 1988; Sutcliffe, 2018; Kent et al., 2021) at finite temperature. In combination with the geodesic nudged elastic band method and the harmonic transition state theory (Bessarab et al., 2012; Bessarab et al., 2015) it furthermore gives insight into the stability of aforementioned objects (Müller et al., 2019).

In this work we introduce the AiiDA-Spirit plugin that connects the Spirit code (Müller et al., 2021) to the AiiDA environment (Huber et al., 2020). AiiDA is an open-source Python framework designed around the FAIR principles of findable, accessible, interoperable and reusable data (Wilkinson et al., 2016) in computational science (Pizzi et al., 2016). Calculations that run through the AiiDA infrastructure are automatically stored as nodes in a database together with all inputs and outputs that are necessary to reproduce the simulation results. This results in an directed acyclic graph that can connect different nodes which can be used to reproduce the data provenance from a final result.

In the context of spin-dynamics simulations, a simulation result could be the magnetic ordering obtained from a minimization of the forces on each spin in an LLG calculation. The outcome of such a simulation will in general depend on input parameters such as the geometry (positions of the spins, size of simulation cell, open or periodic boundary conditions), the exchange coupling constants, or applied external fields as well as temperature noise. But also the starting point for the minimization (e.g., starting from an ordered ferromagnet or from random spin orientations) are important as local minima in the energy landscape can generally be present in which metastable states can be stabilized. To ensure reproducible calculation results, keeping track of the full data provenance of a simulation is necessary.

AiiDA's plugin infrastructure allows to orchestrate and combine different sequences of calculations, possibly using different simulation software and methods, through a common interface. Here, we use this to first generate exchange coupling parameters from DFT calculations using the JuKKR code (The
JuKKR developers, 2021) with the help of the AiiDA-KKR plugin (Rüßmann et al., 2021a; Rüßmann et al., 2021b). Then, we proceed with spin-dynamics simulations using the Spirit code (Müller et al., 2019; Müller et al., 2021) via the newly developed AiiDA-Spirit plugin (The AiiDA-Spirit developers, 2021). This allows to include the full history of the input parameter generation for spin-dynamics calculations in the provenance graph of a Spirit simulation. Using AiiDA therefore facilitates multi-scale modeling that combines the predictive power of DFT calculations and the speed and scalability of spin-dynamics simulations in the same framework.

The AiiDA engine (Uhrin et al., 2021) provides a highly scalable infrastructure that is able to deal with thousands of calculations simultaneously. Together with the simple Python interface that AiiDA-Spirit provides, spin-dynamics simulations are possible in an automated way which can be used in a highthroughput fashion. This opens new possibilities for applying the Spirit code in automated setups and as part of complex workflows in conjunction with other simulation methods such as DFT. This new capability allows to integrate Spirit in the toolbox of methods that are used in automated computational materials design for magnetic materials (Himanen et al., 2019).

This paper is structured as follows. First the methods section introduces the theory behind spin-dynamics simulations. Then the AiiDA-Spirit plugin is presented which is then applied to 1) a parameter exploration based on a toy model and a large number of high-throughput AiiDA-Spirit calculations, 2) to a simple Monte Carlo example to find the critical temperature of a model system, and 3) multi-scale modelling combining DFT and LLG calculations at the example of $\gamma$-Fe. Finally the paper concludes with a discussion of the results.

\section{METHODS}

\subsection{Spirit Theory}

All spin-dynamics simulations shown throughout the paper were performed with the Spirit code (Müller et al., 2019; Müller et al., 2021). The Spirit code provides a framework for atomic-scale spin simulations and combines both a graphical user interface as well as an easy accessible Python API. All simulations performed with Spirit are based on an extended Heisenberg Hamiltonian describing the interaction of spins $\vec{S}_{i}=\vec{M}_{i} / \mu_{i} \quad\left(\mu_{i}=\left|\vec{M}_{i}\right|\right)$ sitting at lattice sites $i$. It can be written in its most general form as

$$
\begin{aligned}
H= & -\sum_{\langle i j\rangle} J_{i j}\left(\vec{S}_{i} \cdot \vec{S}_{j}\right)-\sum_{\langle i j\rangle} \vec{D}_{i j} \cdot\left(\vec{S}_{i} \times \vec{S}_{j}\right) \\
& -\sum_{i} K_{\perp}\left(\vec{S}_{i} \cdot \overrightarrow{\vec{e}}_{z}\right)^{2}-\sum_{i} \mu_{i} \vec{B} \cdot \vec{S}_{i} \\
& -\frac{\mu_{0}}{8 \pi} \sum_{i \neq j} \frac{3\left(\vec{S}_{i} \cdot \overrightarrow{\vec{r}}_{i j}\right)\left(\vec{S}_{j} \cdot \overrightarrow{\vec{r}}_{i j}\right)-\vec{S}_{i} \cdot \vec{S}_{j}}{r_{i j}^{3}} \\
& -\sum_{\langle i j k l\rangle} K_{i j k l}\left(\vec{S}_{i} \cdot \vec{S}_{j}\right)\left(\vec{S}_{k} \cdot \vec{S}_{l}\right) .
\end{aligned}
$$

Here, the first line contains the isotropic and antisymmetric exchange interactions, the later also referred to as Dzyaloshinskii-Moriya 


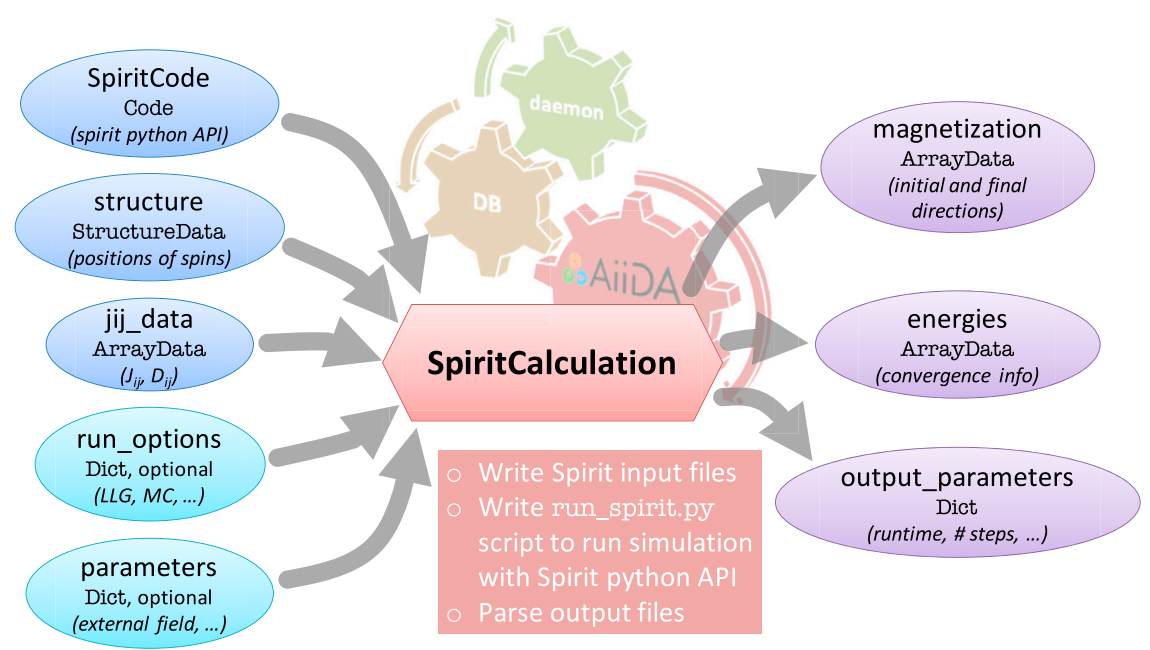

FIGURE 1 | Layout of the SpiritCalculation that is at the heart of the AiiDA-Spirit plugin. On the left hand side the possible input nodes are shown which are translated by the SpiritCalculation into the appropriate input files needed to execute Spirit. The run_options and parameters input nodes are optional that default to a basic LLG calculation starting from random orientation of the spins and without external fields or temperature. The typical output nodes for a LLG calculation are shown on the right hand side.

interaction. The second and third line describe the on-site anisotropy, the Zeeman energy due to an external magnetic field $\vec{B}$, as well as the dipolar contribution. The last term allows to include higher-order exchange interactions (Hoffmann and Blügel, 2020) such as the conventional fourspin or four-spin-four-site interaction (Heinze et al., 2011), the four-spin-three-site interaction (Krönlein et al., 2018), as well as the biquadratic interaction (Szilva et al., 2013). The list of pairs $\langle i j\rangle$ and quadruplets $\langle i j k l\rangle$ as well as their respective parameters $J_{i j}, \vec{D}_{i j}$, and $K_{i j k l}$ can be defined by the user based on the desired use case. Furthermore, the system geometry such as the lattice symmetry and lattice size can be chosen arbitrarily and Spirit allows to introduce defects such as vacancies or atoms of different types. To obtain ground state as well as thermal properties of the investigated system, either the Monte Carlo method based on a Metropolis algorithm or Landau-Lifshitz-Gilbert dynamics can be used.

A more detailed description of the Spirit framework as well as its further functionalities, such as the possibility to calculate lifetimes of magnetic textures based on the combination of geodesic nudged elastic band and harmonic transition state theory calculations, can be found in Ref. (Müller et al., 2019).

\subsection{The AiiDA-Spirit Plugin}

AiiDA's plugin system allows to combine various simulation codes and methods (to date more than 60 plugins exist already (The AiiDA team, 2021)) on the same footing while augmenting the calculation done through the AiiDA infrastructure with the stored data provenance. Albeit their significance in research on magnetic materials, spin-dynamics calculations have not been at the center of the AiiDA community so far. To the best of our knowledge, besides the AiiDA-Spirit plugin presented here, only a first version of the AiiDA-UppASD plugin (Xu et al., 2021) exists for the UppASD code (Skubic et al., 2008) to combine AiiDA with a spin dynamics simulation engine. In the context of AiiDA, a calculation plugin needs to be able to generate typical input files that are required to run a calculation through a bash script that will be generated when a calculation is submitted to a computer or as a job on a supercomputer. At the heart of the AiiDA-Spirit plugin lies the SpiritCalculation that connects the Spirit code via the Spirit Python API to AiiDA. The Layout of the SpiritCalculation is shown in Figure 1. To run a Spirit calculation a number of input nodes are required:

- a structure node describing the lattice of spins (i.e., their positions in the unit cell),

- an array of the corresponding jij_data that contains the pairwise $J_{i j}$ and $\vec{D}_{i j}$ parameters for the extended Heisenberg Hamiltonian (Eq. 1),

- the SpiritCode that is an installation of the Spirit Python API on the computer where the calculation should run,

- and run_options as well as input parameters that control the type of the Spirit run (e.g., LLG or Monte Carlo) or further settings like strength and direction of external fields, respectively.

Additionally, input modes that trigger special features of the Spirit code such as disorder and defects in the structure or pinning of spins to certain directions can be controlled with the corresponding optional input nodes. The SpiritCalculation then implements the functionality to translate this information into the appropriate input files and runs the calculation using the Spirit Python API. The AiiDA daemon automatically takes care of creating a suitable job script, copying necessary input files, and of submitting and monitoring the calculation run. Once the calculation job finishes, important output files are copied back to the retrieved folder in the AiiDA file repository associated to 
the AiiDA database. Then, the SpiritParser extracts useful information that should be stored in the database. For the example of a LLG calculation this entails settings such as the number of LLG steps until convergence, the used wall clock time on the computer where the calcualtion ran, an array of the energies (i.e. exchange energy per spin), and the initial and final directions of the spins in the magnetization array.

Apart from the SpiritCalculation and SpiritParser, AiiDASpirit comes with some tools that can be used in the typical jupyter notebook environment that is often used in the context of AiiDA. In particular we mention the show_spins tool of AiiDASpirit which provides the Spirit visualization capabilities in a simple Python API. This consists of a WebAssembly and WebGL version of the VFRendering library (Vfrendering, 2021) in combination with a JavaScript interface that can be used to visualize the directions of the spins from the web-browser based environment natural to jupyter notebooks.

\subsection{DFT-Based Calculation of Exchange Coupling Constants}

The density functional theory (DFT) results of this work were produced within the generalized gradient approximation (GGAPBE) (Perdew et al., 1996) using the full-potential scalarrelativistic Korringa-Kohn-Rostoker Green's function method (KKR) (Ebert et al., 2011) as implemented in the JuKKR code package (The JuKKR developers, 2021). We use an $\ell_{\max }=3$ cutoff in the angular momentum expansion with an exact description of the atomic cells (Stefanou et al., 1990; Stefanou and Zeller, 1991). After the self-consistent DFT calculations, the method of infinitesimal rotations (Liechtenstein et al., 1987) was used to compute the exchange interaction parameters $J_{i j}$. The series of DFT calculations in this study are orchestrated using the AiiDAKKR (Rüßmann et al., 2021a) plugins to the AiiDA infrastructure (Huber et al., 2020). The complete dataset that includes the full provenance of the calculations is made publicly available in the materials cloud repository (Talirz et al., 2020; Rüßmann et al., 2021).

\section{RESULTS}

\subsection{Automated Landau-Lifshitz-Gilbert Calculations for Model Parameter Exploration}

To illustrate the usage of AiiDA-Spirit we first consider a toy model consisting of a single layer of spins in a simple-cubic lattice. The complete example is part of the dataset that accompanies this publication (Rüßmann et al., 2021). We assume only nearest neighbor interactions with isotropic exchange $J_{1}=10 \mathrm{meV}$ and Dzyaloshinskii-Moriya interactions with a strength of $D_{1}=$ $6 \mathrm{meV}$. This choice of parameters does not reflect any concrete physical system but is chosen for illustration purposes because it is known to produce skyrmions with small radii. The generation of the corresponding input node for the SpiritCalculation where including the directions of the DMI vectors can be seen in the following code snippet.
TABLE 1 | Parameters for the LLG calculations of the toy model. Arrays are indicated by the square brackets. Except for external_field_magnitude and Ilg_temperature all parameters are kept fixed in the simulations.

\begin{tabular}{|c|c|c|}
\hline Parameter & Value & Description \\
\hline n_basis_cells & $(50,50,1)$ & Size of the simulation cell \\
\hline boundary_conditions & (True, True, False) & Periodic boundary conditions \\
\hline llg_n_iterations & 100,000 & Number of iterations \\
\hline llg_damping & 0.3 & Damping constant \\
\hline llg_beta & 0. & Non-adiabatic damping \\
\hline llg_dt & 0.0 & Time step dt (ps) \\
\hline llg_force_convergence & $10^{-7}$ & Force convergence parameter \\
\hline llg_temperature & $0 . .75$ & Temperature (K) \\
\hline external_field_magnitude & $-50 \ldots 50$ & Magnitude of the external field \\
\hline external_field_normal & $(0.0,0.0,1.0)$ & Direction of the external field \\
\hline mu_s & $(2.0)$ & Spin moment $\left(\mu_{B}\right)$ \\
\hline
\end{tabular}

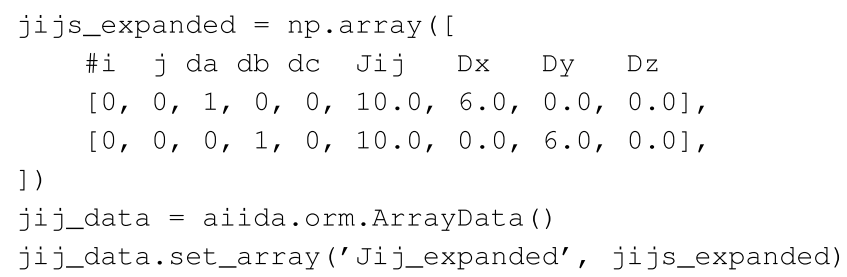

Here $\mathrm{i}, \mathrm{j}$ index the lattice site in the unit cell (situated at $\vec{r}_{i}$, $\vec{r}_{j}$ ) and da, db, dc describe offsets into unit cells further away such that $\vec{R}_{i j}=\vec{r}_{j}-\vec{r}_{i}+d_{a} \vec{a}+d_{b} \vec{b}+d_{c} \vec{c}$ describes the distance between two spins $(\vec{a}, \vec{b}, \vec{c}$ are the Bravais vectors of the lattice), Jij and $\mathrm{Dx}, \mathrm{Dy}, \mathrm{Dz}$ denote the exchange interaction and the three components of the DMI vector. For this example we consider an isolated layer of spins with periodic boundary conditions in the plane. We choose a supercell for the SpiritCalculation of $50 \times 50 \times 1$ spins. Furthermore we apply an external field of various strength (in the code snippet we show the input parameters for a value of $25 \mathrm{~T}$ ) in the direction perpendicular to the film in the following code snippet.

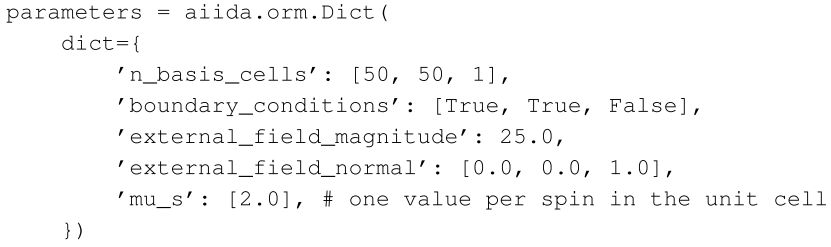

Starting from random orientations of the spins we then perform a time evolution using the LLG method with the Depondt solver (Depondt and Mertens, 2009). The parameters for the LLG calculations are summarized in Table $\mathbf{1 .}$

To harness the high-throughput capabilities of the AiiDASpirit plugin we perform a series of SpiritCalculations to screen a range of external fields and temperatures. We change the temperature from 0 to $75 \mathrm{~K}$ in $2.5 \mathrm{~K}$ steps and vary the external field from $-50 \mathrm{~T}$ to $+50 \mathrm{~T}$ in steps of $2.5 \mathrm{~T}$. The calculations for each parameters set are repeated 5 times starting from different random orientations of the spins for statistical averaging. This amounts to $31 \times 41 \times 5=6,355$ 


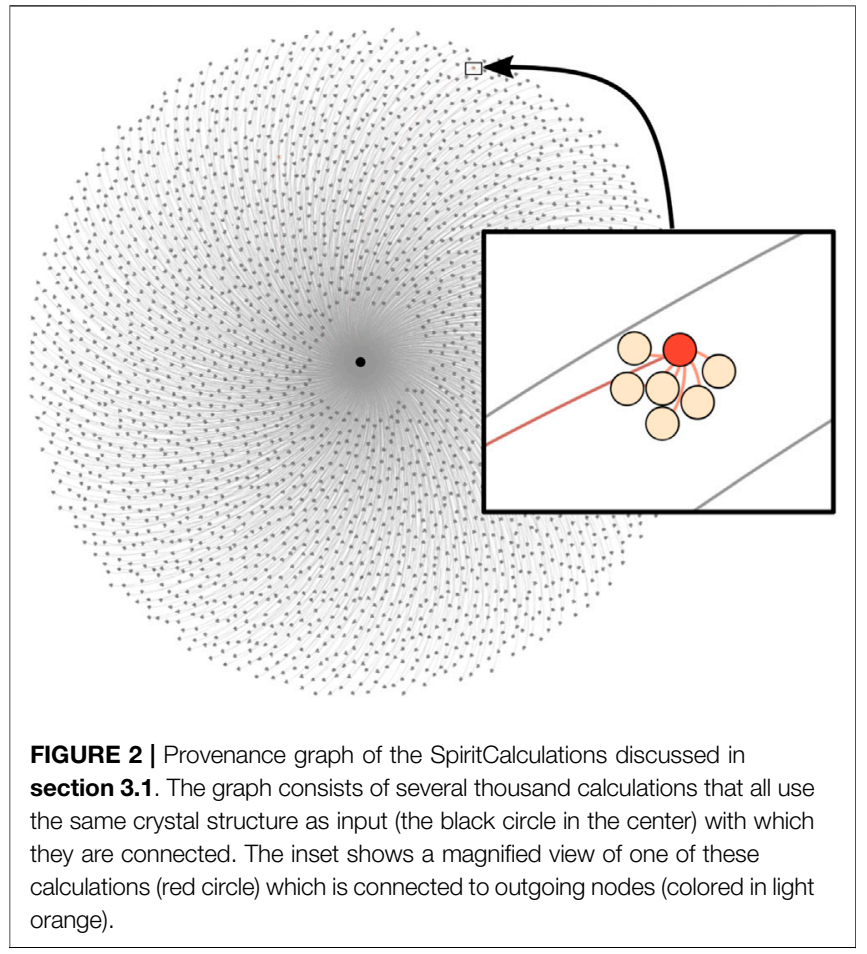

individual SpiritCalculations that were submitted to an in-house compute cluster. We stress that the AiiDA daemon (Uhrin et al., 2021) conveniently takes care of creating submission scripts and automatically retrieves and parses the outcome of the calculations without the need for any user interaction. A visualization of the dataset and the provenance graph for this application is shown in Figure 2.

In order to analyze the outcome of the SpiritCalculations we chose to investigate the topological charge in the simulation cell at the end of the LLG simulation. For a continuous vector field $\vec{m}$ it is defined as

$$
\rho_{\mathrm{T}}=\frac{1}{4 \pi} \int \vec{m} \cdot\left(\frac{\partial \vec{m}}{\partial x} \times \frac{\partial \vec{m}}{\partial y}\right) \mathrm{d} x \mathrm{~d} y .
$$

We added a custom post-processing step to the SpiritCalculation which uses the get_topological_charge function of the spirit Python API. This function calculates the topological charge from the discretized form of Eq. 2 as a summation over all contributions of triangles formed by neighboring spins in the simulation cell (Müller et al., 2019).

Figure 3 shows the outcome of these simulations where the topological charge is shown for all 1,271 pairs $\left(T, B_{z}\right)$ together with selected spin configurations of representative calculations marked by the symbols (Figures 3B-G). The real-space spin configuration at the end of the LLG calculations were visualized using the show_spins tool of the AiiDA-Spirit plugin. It can be seen that a small external field leads to the appearance of skyrmions which in this case have a topological charge of \pm 1 , depending whether they form in a ferromagnetic background of spins pointing in $-z$ (Figure 3C) or $+z$ (Figure 3E) direction. In general, the topological charge counts the difference between

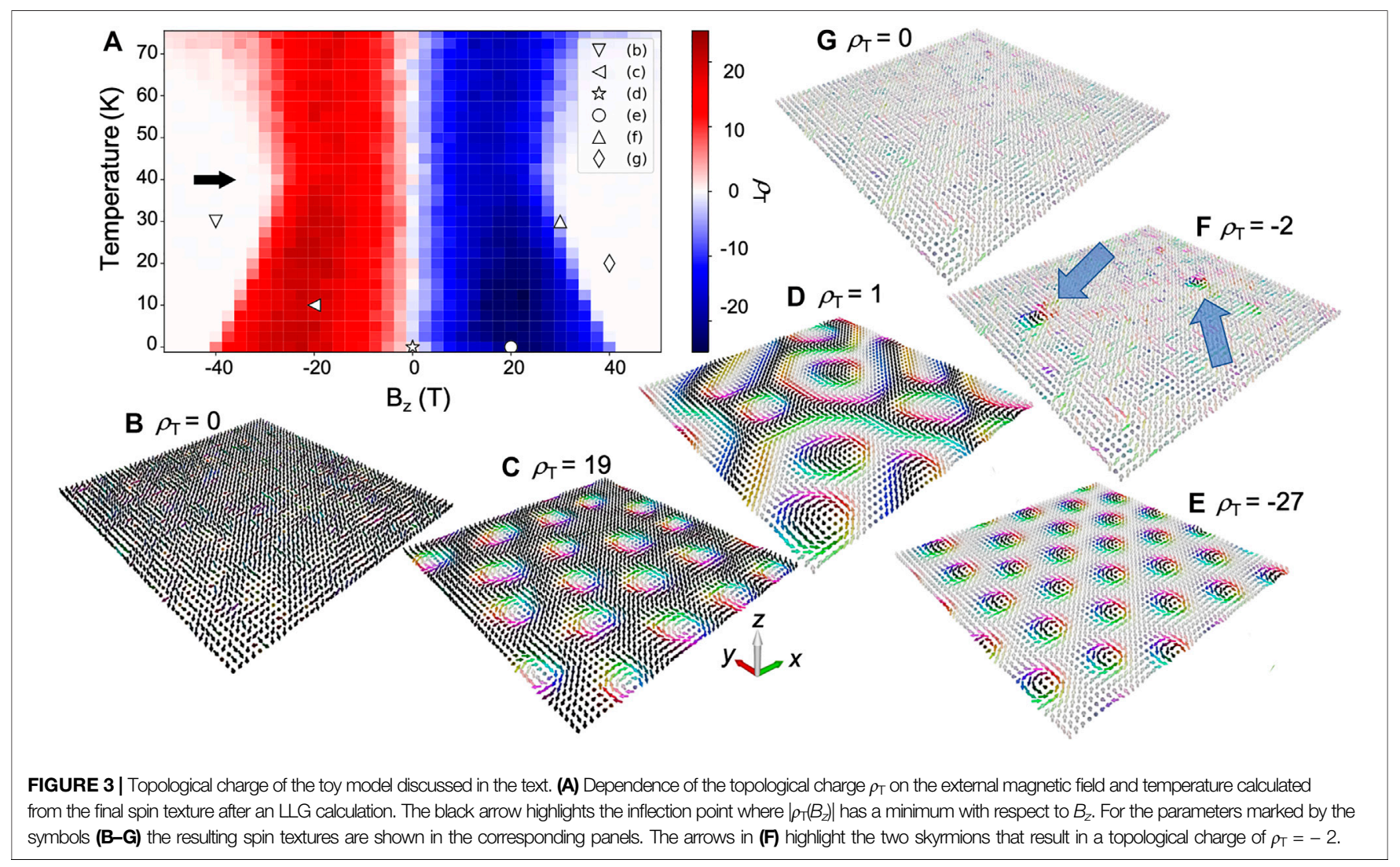


TABLE 2 | Parameters for the MC calculations of the simple-cubic ferromagnet discussed in the text. Note that the chosen settings result in temperature steps of $0.5 \mathrm{~K}$

\begin{tabular}{|c|c|c|}
\hline Parameter & Value & Description \\
\hline n_thermalisation & 5,000 & Number of thermalization steps before n_samples are taken \\
\hline n_samples & 250,000 & Number of samples taken in metropolis algorithm \\
\hline n_decorrelation & 2 & Number of decorrelation steps \\
\hline n_temperatures & 40 & Number of temperature steps \\
\hline T_start & 25 & Start of temperature scanning range \\
\hline T_end & 5 & End of temperature scanning range \\
\hline
\end{tabular}

the amount of skyrmions in up-domains $\left(\rho_{\mathrm{T}}>0\right)$ and skyrmions in down-domains $\left(\rho_{\mathrm{T}}<0\right)$ as seen for vanishing external field in (Figure 3D) where several skyrmions with opposite topological charges lead to a near cancellation of the total topological charge.

At very large fields, the Zeeman exchange coupling term becomes larger than the DMI energy and a homogeneous ferromagnet forms (Figures 3B,G). Temperature fluctuations tend to deform the skyrmions (Figure 3F) and destabilize them. Thus, at elevated temperatures smaller magnitudes of the external magnetic field leads to a vanishing topological charge. As highlighted by the black arrow in (Figure 3A), this is however only true up to a certain critical temperature. For $T>40 \mathrm{~K}$ the topological charge increases again which can be explained by the energy barrier of skyrmion formation and destruction. At these elevated temperatures the fluctuations of the spin directions are larger than $40 \mathrm{~K} \cdot k_{\mathrm{B}} \approx 3.45 \mathrm{meV}$ which we conjecture is the energy barrier for skyrmion formation. While the energy barrier can in principle be calculated by performing geodesic nudged elastic band calculations, this is beyond the scope of this paper and therefore will be omitted. The larger temperature fluctuations also prohibit reaching the force convergence criterion set in the LLG calculation which means that the LLG simulation runs until the maximal simulation time of 100 ps is reached. During this simulation time, skyrmions can spontaneously form and disappear which results in a finite topological charge measured at the end of the run. In the future the real time dynamics of skyrmion creation and collapse may be the focus of the investigation. However, this approach may become unfeasible for situations where the skyrmion lifetime is very long compared to the typical time step in LLG calculations. Finally, we highlight that with increasing temperature fluctuations we also find a larger variance in the number of skyrmions when averaging over the five different starting configuration for each pair $\left(T, B_{z}\right)$. This supports our interpretation that skyrmions are spontaneously created and annihilated by temperature fluctuations.

\subsection{Curie Temperature Using Monte Carlo}

The Monte Carlo (MC) method is a well established tool in physics which, when applied to spin systems, allows to estimate the critical temperature of the magnetic ordering (Curie temperature) (Binder and Heermann, 1997). The Spirit code (Müller et al., 2019) implements a Metropolis

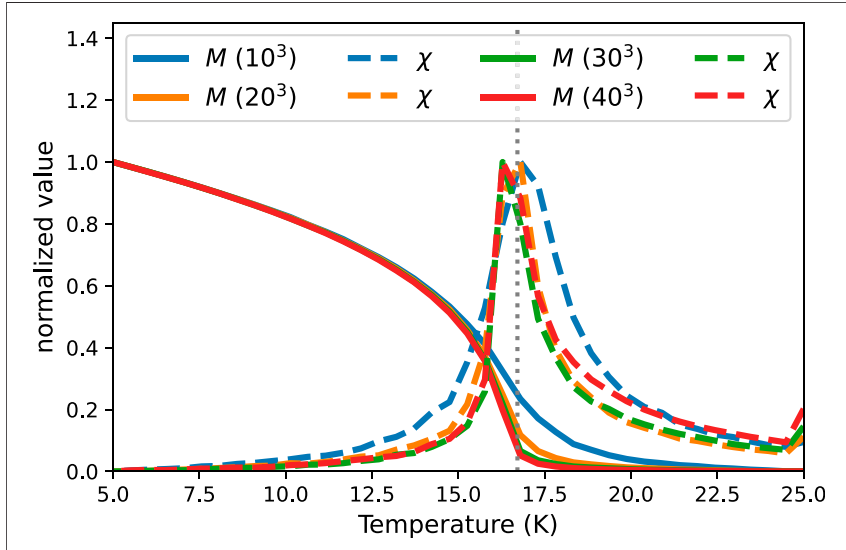

FIGURE 4 | Results of the Monte Carlo calculations for a simple-cubic ferromagnetic with nearest neighbor $J_{1}=1 \mathrm{meV}$ exchange interactions. Shown are results for $10 \times 10 \times 10$ to $40 \times 40 \times 40$ supercells where the solid lines show the normalized value of the total magnetization $M$ and the dashed lines the corresponding susceptibility $\chi$. The dashed vertical line indicate the expected value of the critical temperature at $T_{C}=16.71 \mathrm{~K}$.

algorithm which can be used from AiiDA-Spirit by choosing the mc simulation method (instead of the previously used LLG method). We demonstrate the MC at the example of a simple-cubic ferromagnet with only nearest neighbor interactions $J_{1}=1 \mathrm{meV}$. We perform calculations for varying supercell sizes between $10 \times 10 \times 10$ and $40 \times$ $40 \times 40$ with the MC parameters given in Table 2 . The results of the calculation are shown in Figure 4 where, together with the total magnetization $M$, the isothermal susceptibility

$$
\chi=\frac{1}{k_{\mathrm{B}} T}\left(\left\langle M^{2}\right\rangle-\langle M\rangle^{2}\right)
$$

with $M=\left|\frac{1}{N} \sum_{i} \vec{S}_{i}\right|$ the average magnetization of the sample is shown. We see that $M(T)$ converges with increasing supercell size indicating that boundary effects become less important. The corresponding susceptibilities show a diverging behavior at $T_{c}$. Our calculation results agree well with the expected value of $T_{c}=$ $1.44 J_{1} / k_{\mathrm{B}}=16.71 \mathrm{~K}$. We stress that these calculations require a series of steps consisting of, for example, thermalization and decorrelation steps at each temperature value in the scanning interval. Within AiiDA-Spirit this complexity is conveniently absorbed in the SpiritCalculation which greatly facilitates the application of MC calculations. 
A

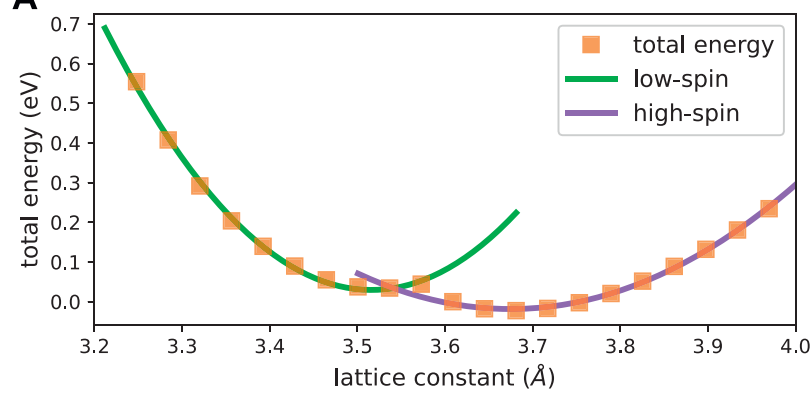

C

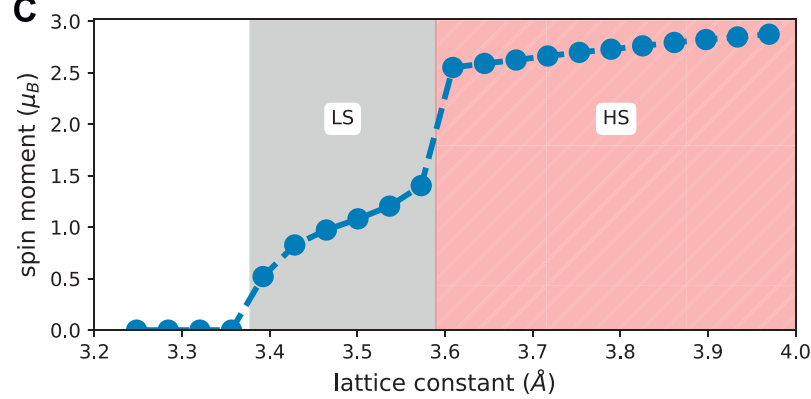

B

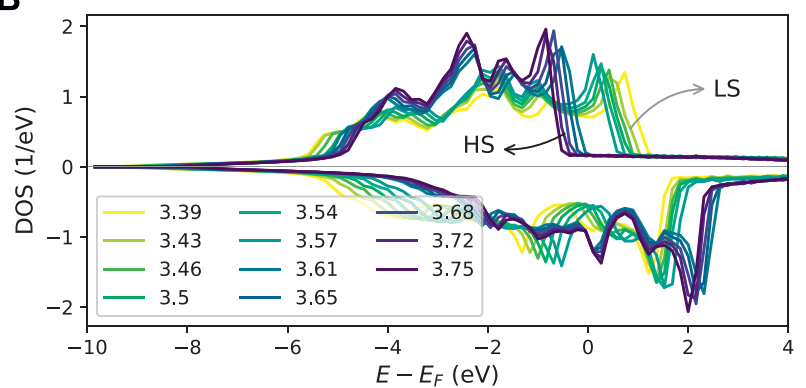

D

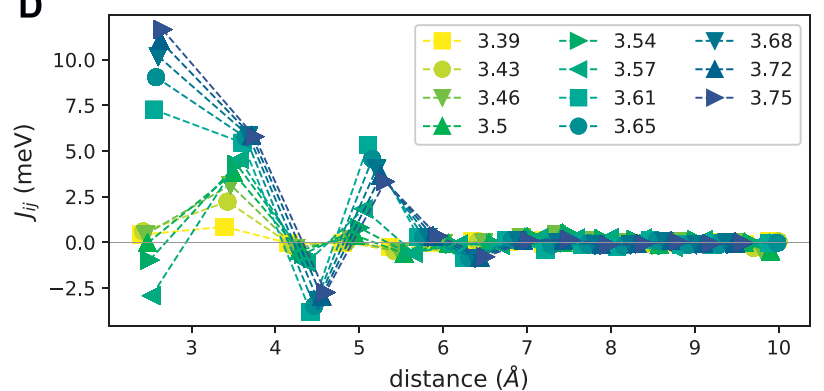

FIGURE 5 | Results of the DFT calculations for $\gamma$-Fe. Total energy as a function of the lattice constant (A) where the green and violet lines show parabolic fits to lowspin $\left(a_{\text {lat }}<3.6 \AA\right)$ and high-spin $\left(a_{\text {lat }} \geq 3.6 \AA\right.$ ) states. (B) Corresponding density of states (value in the legend indicates the lattice constant) which clearly separates into low-spin (LS) with smaller exchange splitting and high-spin (HS) states with larger exchange splitting. Note that the positive (negative) values of the DOS indicate the majority (minority) spin channels. (C) Spin moment as a function of the lattice constant where the transition from non-magnetic (first four data points) over the low-spin state to the high-spin state is evident. (D) Resulting exchange coupling constants $\left(J_{i j}\right)$ as a function of the pairwise distance between the Fe atoms in $\gamma$-Fe (the lattice constant is given in the legend).

\subsection{Multi-Scale Modeling: $\gamma$-Fe}

We now demonstrate how the integration of the Spirit code into the AiiDA framework through the AiiDA-Spirit plugin can facilitate multi-scale modeling for magnetic materials. In this example we first calculate the exchange interaction parameters for $\gamma$-Fe using density functional theory which are then passed to the AiiDA-Spirit plugin for LLG simulations.

The $\gamma$ phase of $\mathrm{Fe}$ is a metastable high-temperature phase where the atoms crystallize in the fcc lattice (Knöpfle et al., 2000; Sjöstedt and Nordström, 2002). This has a drastic consequence on the exchange interactions where, in contrast to the ferromagnetic $\mathrm{bcc} F e$, frustrated exchange interactions can lead to the formation of spin-spirals. Experimentally this structure of Fe can be realized in a Cu matrix (Tsunoda, 1989; Tsunoda et al., 1993). It is known that a variation of the lattice constant of $\gamma$-Fe can have drastic consequences for the magnetic ordering (Sjöstedt and Nordström, 2002). Here, we investigate bulk crystals of $\gamma-\mathrm{Fe}$ for varying lattice constants between $a_{\text {lat }}=3.2 \AA$ and $a_{\text {lat }}=4.0 \AA$ around the lattice constant of $\mathrm{Cu}\left(a_{\text {lat }}=3.6 \AA\right)$.

Figure 5 summarizes the results of the DFT calculations that were done with the AiiDA-KKR plugin (see methods section for numerical details). The total energy as a function of the lattice constant (shown in panel Figure 5A) reveals a phase transition from the low-spin state (for $a_{\text {lat }}<3.6 \AA$ ) to the high-spin state $\left(a_{\text {lat }} \geq 3.6 \AA\right)$ of $\gamma$-Fe as seen in the jump of the spin moment to $\mu_{s}>2.5 \mu_{B}$. This coincides with a smaller exchange splitting seen in the density of states (Figure 5B) and consequently a smaller value of the spin moment (Figure 5C). For lattice constants below
$3.37 \AA$ we find that the magnetic moment vanishes. Panel (Figure 5D) shows the calculated exchange interactions $J_{i j}$ as a function of distance between two Fe atoms. Clearly, the sign of the nearest neighbor interaction shows the most drastic change with the transition from high-spin to low-spin state at smaller lattice constant of $\gamma$-Fe. While in the high-spin state the first and second nearest neighbor interaction are both ferromagnetic $\left(J_{i j}>0\right)$, for the low-spin state the nearest neighbor interaction changes from being weakly ferromagnetic to antiferromagnetic $\left(J_{i j}<0\right)$.

In the following, the consequences of this change for the magnetic ordering are investigated based on a series of LLG calculations using the AiiDA-Spirit plugin. In the DFT calculation we use the primitive cell which contains a single atom in the unit cell. For the spirit calculations we map the calculated exchange interactions onto the conventional unit cell consisting of four atoms. The parameters of the LLG simulations are summarized in Table 3 . We study the magnetic ordering in a $40 \times 40 \times 40 \times 4=256,000$ spins supercell without external magnetic fields and at temperature $T=0 \mathrm{~K}$. Here we focus on the ground state that forms and therefore neglect effects of temperature fluctuations and external fields which can, for example if $T$ is high enough, overcome the energy barrier between different (metastable) magnetic orderings. We further neglect the influence of anisotropy $\left(K_{\perp}=0\right)$ in this work and we also do not include higher order exchange terms $\left(K_{i, j, k, l}=0\right)$. We choose open boundary conditions in order to not bias the eventually resulting spin-spiral wavelength by the periodicity of the supercell. Table $\mathbf{4}$ summarizes the DFT calculated 
TABLE 3|Parameters for the LLG calculations for $\gamma$-Fe. Note that the simulation cell consists of $40 \times 40 \times 40 \times 4=256,000$ atoms due to the choice of the conventional unit cell with four atoms. The spin moment $\mu$ is extracted from the DFT calculation at the respective lattice constant and open boundary conditions are chosen. Parameters not listed here are set to the same value as in Table $\mathbf{1 .}$

\begin{tabular}{|c|c|c|}
\hline Parameter & Value & Description \\
\hline n_basis_cells & $(40,40,40)$ & Size of the simulation cell \\
\hline boundary_conditions & (False, False, False) & Open boundary conditions \\
\hline \|lg_temperature & 0 & Temperature (K) \\
\hline external_field_magnitude & 0 & Magnitude of the external field \\
\hline mu_s & $(\mu, \mu, \mu, \mu)$ & Spin moment $\left(\mu_{B}\right)$ \\
\hline
\end{tabular}

TABLE 4 | Input parameters extracted from DFT that are used in the SpiritCalculations for $\gamma$-Fe for different lattice constants $a_{\text {lat }}$ (given in $\AA$ ). Listed are the magnetic moment $\mu$ (in $\mu_{B}$ per spin) and the exchange coupling parameters $J_{i j}$ for the first seven shells (denoted $J_{1}$ to $J_{7}$ ) which are given in meV.

\begin{tabular}{ccccccccc}
$\boldsymbol{a}_{\text {lat }}$ & $\boldsymbol{\mu}$ & $\boldsymbol{J}_{\mathbf{1}}$ & $\boldsymbol{J}_{\mathbf{2}}$ & $\boldsymbol{J}_{\mathbf{3}}$ & $\boldsymbol{J}_{\mathbf{4}}$ & $\boldsymbol{J}_{\mathbf{5}}$ & $\boldsymbol{J}_{\mathbf{6}}$ & $\boldsymbol{J}_{\mathbf{7}}$ \\
\hline 3.39 & 0.52 & 0.42 & 0.85 & -0.04 & -0.03 & -0.27 & 0.00 & 0.05 \\
3.43 & 0.83 & 0.60 & 2.23 & -0.17 & 0.03 & -0.48 & 0.00 & 0.04 \\
3.46 & 0.97 & 0.42 & 3.15 & -0.31 & 0.16 & -0.57 & -0.04 & 0.00 \\
3.50 & 1.08 & -0.05 & 3.79 & -0.45 & 0.38 & -0.61 & -0.07 & -0.04 \\
3.54 & 1.20 & -0.98 & 4.28 & -0.64 & 0.80 & -0.62 & -0.11 & -0.10 \\
3.57 & 1.40 & -2.92 & 4.56 & -1.11 & 1.83 & -0.59 & -0.09 & -0.16 \\
3.61 & 2.55 & 7.27 & 5.46 & -3.82 & 5.33 & 0.29 & -0.87 & 0.12 \\
3.65 & 2.59 & 9.05 & 5.75 & -3.48 & 4.57 & 0.29 & -0.87 & 0.12 \\
3.68 & 2.62 & 10.19 & 5.85 & -3.19 & 4.05 & 0.30 & -0.86 & 0.12 \\
3.72 & 2.66 & 11.03 & 5.84 & -2.96 & 3.67 & 0.32 & -0.83 & 0.11 \\
3.75 & 2.70 & 11.66 & 5.79 & -2.75 & 3.33 & 0.35 & -0.80 & 0.10 \\
\hline
\end{tabular}

values for magnetic moments and exchange coupling constants for varying lattice constants that were used in the respective SpiritCalculations. Note that the exchange coupling constants are only shown up to the seventh shell of neighbors but the calculations included pairs up to the 15 th shell that are however much smaller than the values reported in Table 4.

We start the discussion of the LLG calculations with the results for $\gamma$-Fe in the lattice constant of $\mathrm{Cu}\left(a_{\mathrm{lat}}=3.61 \AA\right)$. Figure 6A shows the resulting spin texture at the end of the LLG calculation for the central layer of spins in the $y z$-plane (shown in the inset Figure 6B). We can see that a spin-spiral forms in $z$-direction with ferromagnetically ordered spins in $y$ direction. At the open boundaries we see that the missing neighbors on one side influence the magnetic ordering that deviates from the spin-spiral in the center for a distance of about five lattice constants. In order to quantify the spin-spiral we pick the two cardinal directions in this plane (indicated by blue and orange lines in Figure 5A) and extract the $z$ component of the spin $S_{z}$. We combine the projections onto the $y z$-plane from two adjacent layers of spins (indicated by the two grey planes in Figure 5B) to not restrict our analysis to a single sub-lattice only. This allows to describe also antiferromagnetic structures in the sub-lattices with ferromagnetic ordering within one sub-lattice, which will be important later. Figure 6C shows that, except for boundary effects, $S_{z}$ stays constants when following the $y$-direction. Along the $z$-axis we see a complex beating pattern with the site index that can be decomposed into two $\pi / 2$-shifted spin- spirals in the two different sub-lattices. The corresponding Fourier transformation

$$
\mathcal{F}_{j}(q)=\frac{1}{\sqrt{2 \pi}} \int e^{-i q r_{j}} S_{z}\left(r_{j}\right) \mathrm{d} r_{j}
$$

with $j=y, z$ computed with the fast Fourier transform algorithm (FFT) is shown in (Figure 6D). As expected, the FFT of the predominantly ferromagnetically ordered spins along the $y$ direction $\left|\mathcal{F}_{y}\right|$ mainly shows a signal at $q=0$ whereas $\left|\mathcal{F}_{z}\right|$ shows the appearance of four peaks at $q \approx \pm 0.2 \frac{2 \pi}{a_{\mathrm{lat}}}$ and $q \approx \pm 0.8 \frac{2 \pi}{a_{\text {lat }}}=(1-0.2) 2 \pi / a_{\text {lat }}$ which are attributed to the two $\pi / 2$-shifted oscillations in the two sub-lattices. We point out that the reflection symmetry around $q=0$ is a consequence of the realvalued input to the FFT and is therefore not discussed further. As seen from the FFT in $y$-direction and from the corresponding spin texture in (Figure 6A) the spins in the direction perpendicular to the propagation direction of the spin spiral (i.e., the $z$-direction in this example) are ordered ferromagnetically. Therefore the spin-spiral wavevector is $\vec{q}=(0,0,0.2) 2 \pi / a_{\text {lat }}$. After having characterized the spin-spiral ground state of $\gamma$-Fe we continue with a discussion of the magnetic ordering depending on the changing exchange coupling parameters with changing lattice constant.

Figure 7 summarizes the LLG calculations for $\gamma$-Fe for varying lattice constants using the respective set of exchange parameters shown in Figure 5D. The lines in Figure 7A show the energy at the end of the LLG calculation starting either from a random spin configuration ( $E$, dashed orange) or from the ferromagnetic ( $E_{\mathrm{FM}}$, solid blue) state. We find that for lattice constants $a_{\text {lat }} \geq 3.65 \AA$ the ferromagnetic state minimizes the energy $\left(E-E_{\mathrm{FM}}=0\right)$. We attribute this to the increasing ferromagnetic interaction for nearest neighbor spins in the high-spin state which was discussed with Figure 5D. At smaller lattice constants $\left(a_{\text {lat }} \leq 3.6 \AA\right)$, the ferromagnet (FM) is not the ground state anymore. Here we find either a spin-spiral (SS) ground state or an antiferromagnetic (AFM) phase. Figure 7C shows three representative images of the SS, AFM and FM states.

In the SS state the magnetization rotates from left to right (i.e. along the $y$-axis) and shows antiparallel alignment of the rows in $z$-direction. Along the $x$-axis (direction perpendicular to the drawn plane) the spins are aligned ferromagnetically, except for boundary effects at the open ends of the simulation cell (seen in the direction of the first layer of spins). In $z$-direction, adjacent layers are antiferromagnetically ordered. Thus the spin-spiral wavevector for these lattice constants has the 

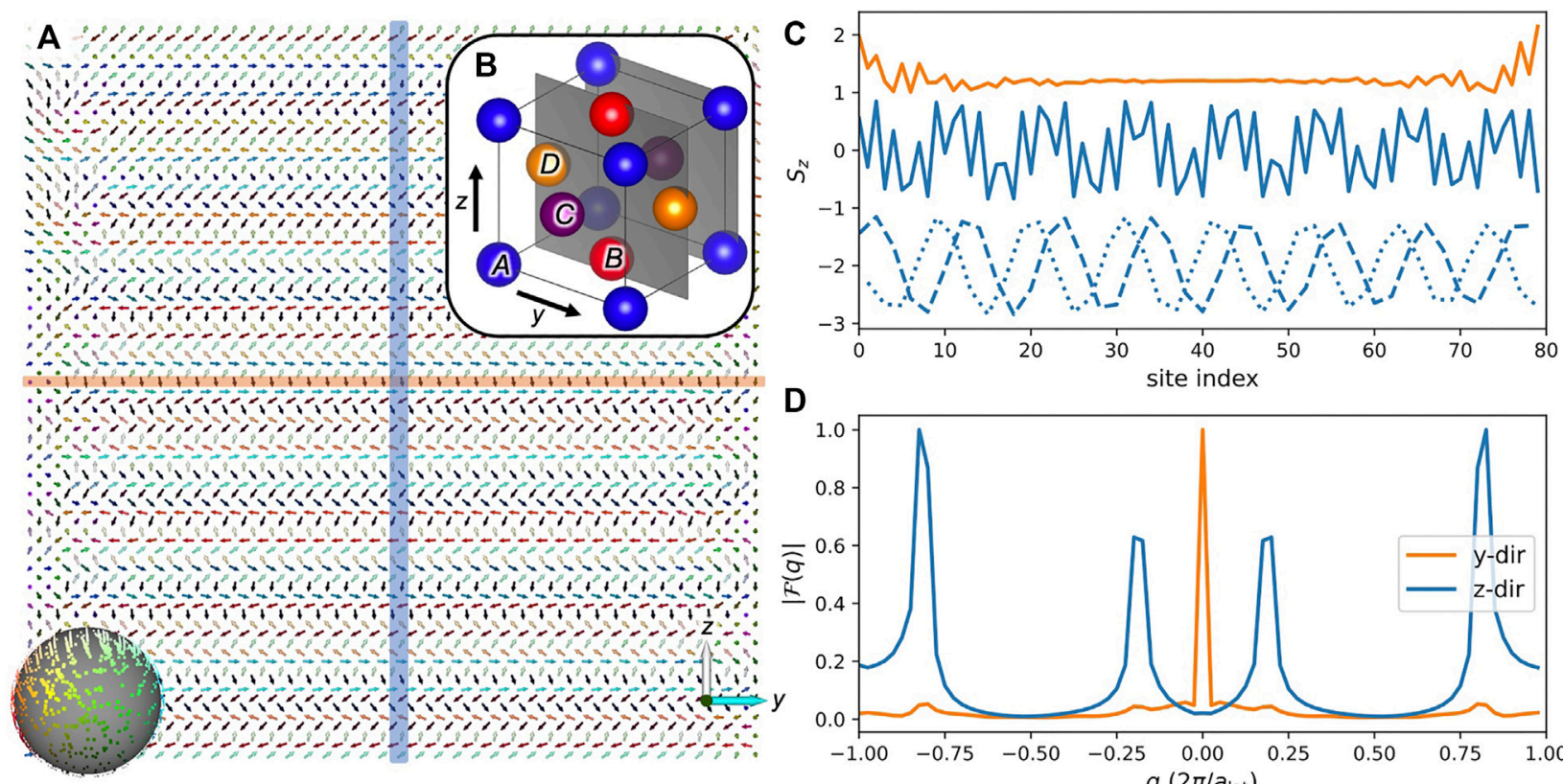

D

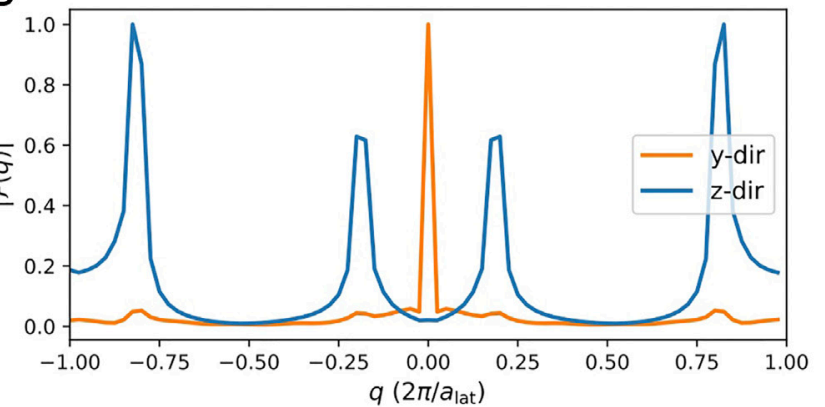

FIGURE 6 | Spin-spiral ground state for $\gamma$-Fe at a lattice constant of $a_{\text {lat }}=3.61 \AA$. (A) Cut through the central $y z$-plane of the $40 \times 40 \times 40$ supercell. Each unit cell consists of 4 Fe atoms on the sub-lattices $A, B, C$ and $D$ shown in the inset (B). The colored points on the grey sphere in the lower left of panel (A) show a projection of the direction of the spins onto the unit sphere. (C) $z$-component of the spin along the $y$ - (orange line) and $z$-directions (solid blue line) indicated in (A) with the corresponding colored stripes. Each direction combines the spins from two adjacent $y z$-planes to cover more than a single sub-lattice per direction (i.e. sites in $y$-direction consist of atoms $A-B-A-\ldots$.$) . The dashed and dotted lines in (C) are a decomposition of S_{z}(z)$ into the sub-lattices $A$ and $D$. Note that the orange and dashed and dotted blue lines are shifted by \pm 2 with respect to the solid blue line. (D) Magnitude of the Fourier transformation of $S_{z}$ along the $y$ - and $z$-directions, i.e. solid orange and blue lines in (C), respectively.

form $\vec{q}=(0, q, 1) 2 \pi / a_{\text {lat }}$ which due to the cubic symmetry of the crystal is equivalent to $\vec{q}=(q, 0,1) 2 \pi / a_{\text {lat }}$. Note that $\vec{q}_{\mathrm{AF}}=$ $(0,0,1) 2 \pi / a_{\text {lat }}$ is the antiferromagnet because the distance between two layers in the $(0,0,1)$ direction of the fcc lattice is $a_{\text {lat }} / 2$.

In the AFM phase the direction of the spins separate into four sub-lattices that correspond to the four atoms in the conventional fcc unit cell. Within each sub-lattice the spins are aligned parallel and form a right angle with their neighboring spins from different sub-lattices. This is highlighted with a red box in the middle panel of (Figure 7C). In the FM phase (lower panel) all spins point in the same direction. Note that in all these calculations the spins can collectively rotate since we neglected contributions from single-ion anisotropies and do not apply an external field.

The summed magnitude of the Fourier transform along the three cardinal axes

$$
|\mathcal{F}(q)|=\sum_{j=x, y, z}\left|\mathcal{F}_{j}(q)\right|
$$

is shown in Figure 7B for different lattice constants. Note that we have summed here over the symmetry-equivalent directions along the $x$ - $y$ - and $z$-directions because of the rotational invariance of the complete spin-structure. Starting from the smallest lattice constant of $a_{\text {lat }}=3.39 \AA$ we see a peak in $|\mathcal{F}(q)|$ at $\vec{q}=( \pm 0.195,0,1) 2 \pi / a_{\text {lat }}$ which corresponds to a wavelength of the spin-spiral of $5.13 a_{\text {lat }}$. Here we focus our discussion on the peak at smaller $q$ values as the same arguments hold for the second peak at $2 \pi / a_{\text {lat }}-q$ as discussed above. With increasing lattice constant the spin-spiral wavelength increases to $7.82 a_{\text {lat }} / 2\left(\vec{q}=( \pm 0.128,0,1) 2 \pi / a_{\text {lat }}\right)$ at a lattice constant of $a_{\text {lat }}=3.5 \AA$. For $3.5 \AA<a_{\text {lat }}<3.6 \AA$ the AFM state is found, which in the Fourier transform is characterized by the dominating peak at $q= \pm 2 \pi / a_{\text {lat }}$. Note that we still get a considerable signal at $q=0$ because we sum over all three cardinal directions and there is ferromagnetic ordering along one direction (see Figure 7C). As discussed above, for larger lattice constants the spin-spiral state briefly shows up again at $a_{\text {lat }}=3.61 \AA$ with a wavelength of $5.59 a_{\text {lat }}$ which however has ferromagnetically ordered spins in both directions perpendicular to the direction of spin-spiral propagation $(\vec{q}=$ $\left.( \pm 0.179,0,0) 2 \pi / a_{\text {lat }}\right)$ until for $a_{\text {lat }} \geq 3.65 \AA$ the FM state is found which only shows a significant Fourier amplitude at $q=0$.

The appearance of the AFM phase for $3.5 \AA<a_{\text {lat }}<3.6 \AA$ can be attributed to the sign change of the nearest neighbor interaction from ferro- to antiferromagnetic. To verify this hypothesis we employ a series of LLG calculations through AiiDA-Spirit. We chose to start from the setup of the calculation for $a_{\text {lat }}=3.61 \AA$, which was found to reproduce the spin-spiral phase. We then modify the strength of the nearest neighbor interaction $J_{1}$ ranging from $-5 \mathrm{meV}$ to + $15 \mathrm{meV}$ and run LLG calculations starting from the FM state, from the AFM state, from the SS phase and random spin orientations. For the AFM state we construct the row-wise AFM orientation of the spins. All other parameters for the LLG calculation are kept constant. In total this is another set of 84 

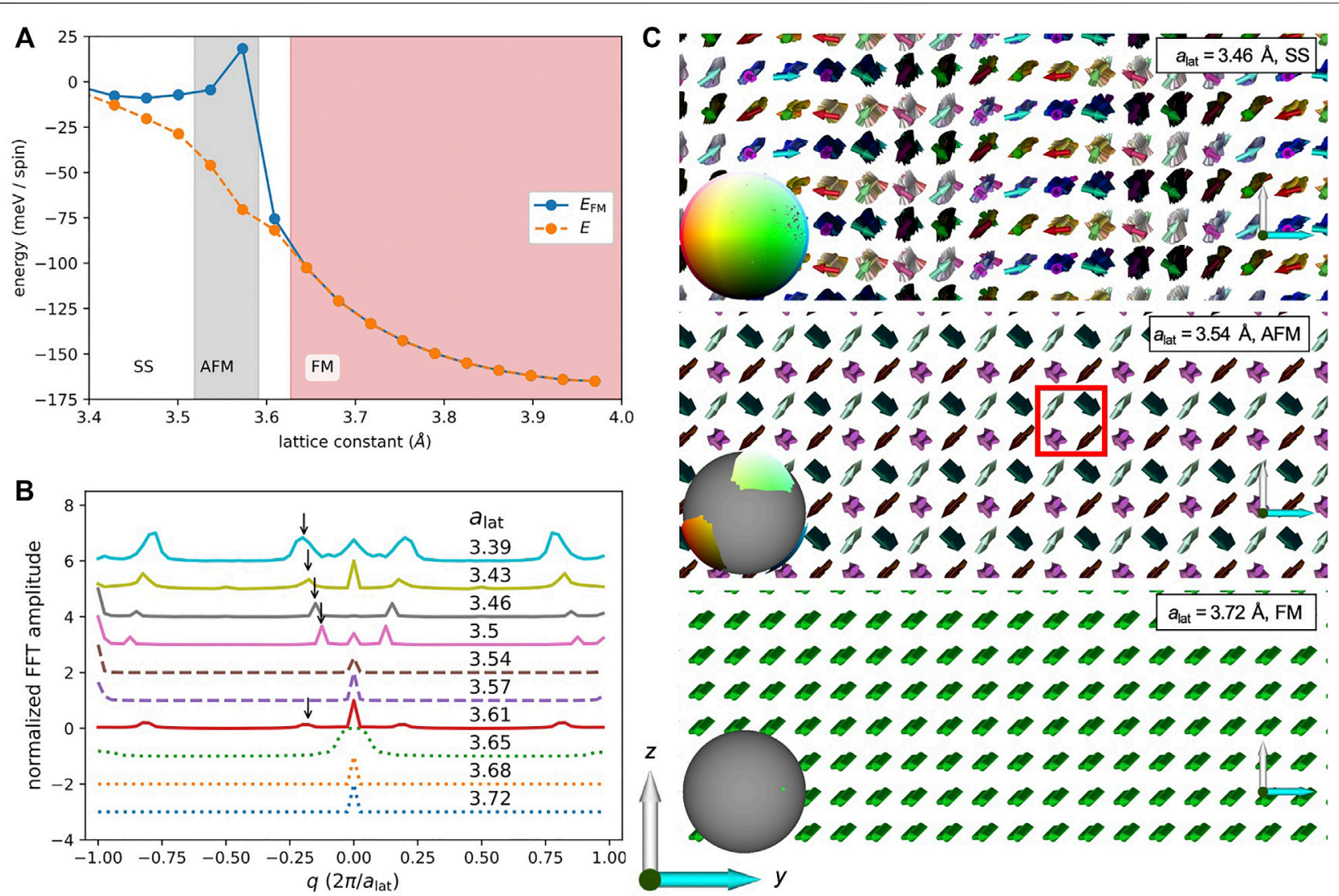

FIGURE 7 | Magnetic ground state in $\gamma$-Fe from spin-dynamics simulations via the AiiDA-Spirit plugin. (A) Calculated energies per spin of the final state after an LLG calculation. Each data point uses the exchange constants computed from DFT (cf. Figure 5D). The solid blue and dashed orange lines indicate the energy computed starting from random spin configuration or the ferromagentic (FM) state. The white, grey and red shaded areas indicate if, respectively, a spin-spiral (SS), antiferromagnetic (AFM) or FM ordering is found to be the ground state. (B) Normalized Fourier transform of the $z$-component of the magnetization in the $y z$-plane for different lattice constants, shifted for clarity. Solid lines correspond to SS, dashed lines to AFM and dotted lines to FM solutions, respectively. The arrows highlight the principal wavenumber of the spin-spiral and highlight their change with the lattice constant. (C) Visualization of representative spin structures for (from top to bottom) SS, AFM and FM states where the red box in the AFM structure highlights a unit cell with the four sub-lattices. The colored points on the grey sphere in the lower left panels show a projection of the direction of the spins onto the unit sphere.

SpiritCalculations where we find that starting from a random spin configuration coincides with starting from the SS. The random starting point is therefore omitted in the following discussion. Figures 8A,B show the dependence of the energy per spin at the end of the LLG calculations. We point out that the LLG calculations for the FM and AFM states are converged in the very first iteration which indicates that the FM and AFM phases are local minima in the energy landscape. We find that the SS state is lowest in energy with a maximal energy gain of $\sim$ $7 \mathrm{meV} / \mathrm{spin}$ in the transition region where $E_{\mathrm{FM}}-E_{\mathrm{AFM}}$ changes sign. From the final spin structure of the spin-spiral solution we proceed with an analysis of the Fourier components as introduced in equations 4 and 5. This is shown in Figure 8C. As highlighted by the grey line, we see an increase in the spinspiral wavevector with increasing $J_{1}$ up to the point where $E_{\mathrm{FM}}$ and $E_{\mathrm{AFM}}$ cross around $J_{1}=7.3 \mathrm{meV}$. As in the previous analysis for changing lattice constant we find that the spin-spiral state is characterized by two wavevectors at $q$ and $2 \pi / a_{\text {lat }}-q$ (grey dashed line). Furthermore, the Fourier transform for all states show significant signals at $q=0$ (indicating parallel spins) and $q=$ $\pm 2 \pi / a_{\text {lat }}$ (indicating antiparallel spins).

Overall we can conclude that the resulting spin-texture in the 256,000 spin unit cell with open boundary conditions is a result of the complex competition of distance-dependent exchange couplings that favor ferromagnetic or antiferromagnetic alignments of spins or can compete and stabilize spin-spiral ground states.

\section{DISCUSSION}

In this article we have presented the AiiDA-Spirit plugin that connects the spin-dynamics code Spirit to the AiiDA framework. AiiDA enables high-throughput calculations while automatically keeping track of the data provenance (Huber et al., 2020). We have demonstrated the capabilities of the AiiDA-Spirit plugin with three examples; 1) high-throughput spin-dynamics calculations based on the Landau-Lifshitz-Gilbert (LLG) 

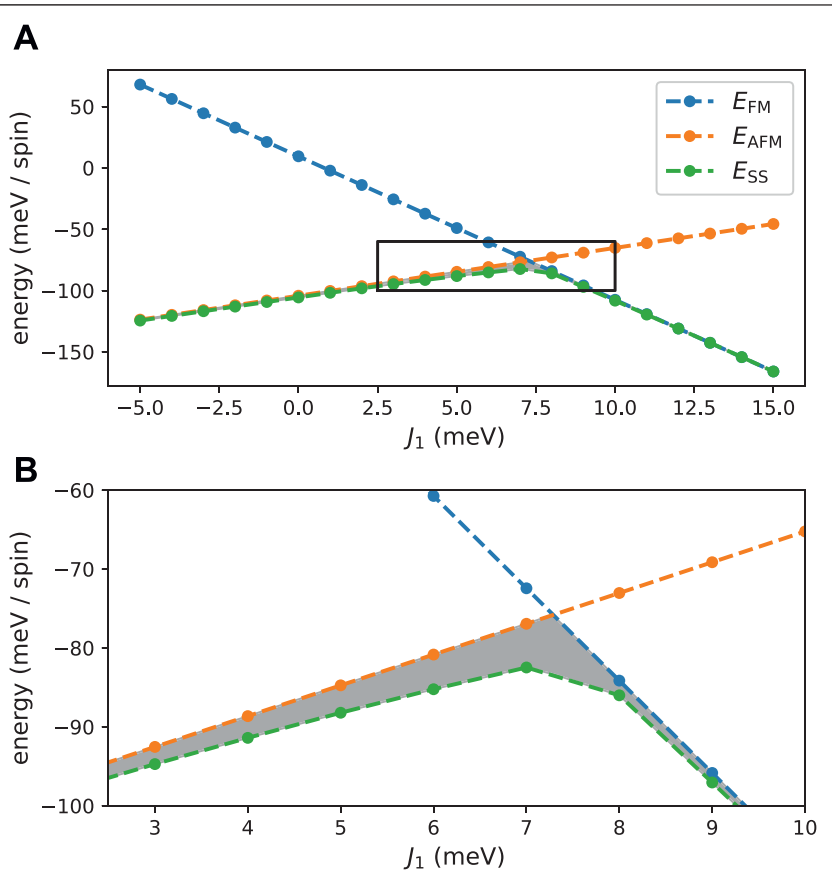

C

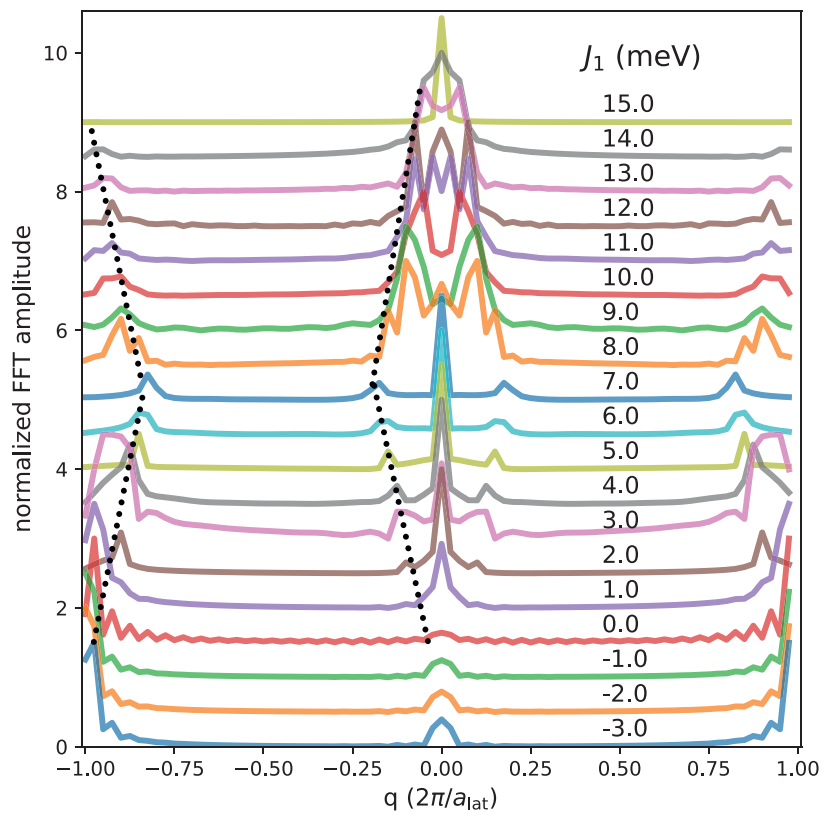

FIGURE 8 | (A) Spin-spiral (SS) energies as a function of the nearest neighbor interaction $J_{1}$ in comparison to ferromagnetic (FM) and antiferromagnetic (AFM) states. Exchange coupling constants were taken from the $a_{\text {lat }}=3.61 \AA$ calculation and the nearest neighbor couplings $J_{1}$ were $m o d i f i e d$ in the range from -5 to 15 meV. (B) Zoom into the transition region marked by the black box in (A). The grey area highlights the energy gain in the SS state compared to FM or AFM states. (C) Normalized Fourier transform of the $z$-component of the magnetization in the $y z$-plane. The spectra are shifted for clarity. Dashed lines indicate where $E_{\mathrm{AFM}}-E_{\mathrm{SS}}<2$ meV and dotted lines are used for $E_{\mathrm{FM}}-E_{\mathrm{SS}}<2 \mathrm{meV}$. The solid and dashed grey lines are guides to the eye highlighting the change in the spin-spiral wavevector with $J_{1}$.

equation for a toy model that shows skyrmions, 2) Monte Carlo calculations for finding the critical temperature of a simple-cubic model ferromagnet, and 3) multi-scale modelling combining density functional calculations with spin-dynamics simulations for $\gamma$-Fe.

In our high-throughput LLG calculations we performed more than 6,000 simulations of a model system consisting of a $2 \mathrm{D}$ lattice of spins in the simple-cubic lattice. The model parameters were chosen such that topologically nontrivial skyrmions appear in the magnetic textures. We varied the temperature and the external magnetic field as external parameters and investigate the change in the topological charge, which is a measure of the number of skyrmions that appear in the system. We find that, starting from $T=0$, the transition to the homogeneous ferromagnetic phase happens at lower magnetic fields. At a certain critical temperature however the number of skyrmions starts increasing again. We interpret this as the surpassing of the energy barrier for skyrmion formation which can be overcome by temperature fluctuations of the spins. This goes hand in hand with a larger variance in the topological charge that we measure from averaging multiple runs for each pair of $\left(T, B_{z}\right)$. These calculations demonstrate the possibility to employ the AiiDASpirit plugin for high-throughput spin-dynamics simulations which make parameter exploration easier accessible.

In our Monte Carlo calculations we showed how the complex series of calculations necessary for finding the ordering temperature of a simple-cubic ferromagnet (several calculations across the transition region from ferromagnetically ordered to paramagnetic state have to be performed) can be found from a single SpiritCalculation of the AiiDA-Spirit plugin. Our simulation result is in good agreement with the theoretically expected result. The ease-of-use for these calculations facilitate the incorporation of AiiDA-Spirit calculations in complex workflows in materials informatics for magnetic materials. Here, finding the critical temperature of a magnetic material is a very common problem.

Finally, we discussed the use case of LLG calculations for the study of the magnetic ordering of $\gamma$-Fe, which is the hightemperature fcc phase of $\mathrm{Fe}$. From experiments, where $\mathrm{Fe}$ clusters were embedded in a $\mathrm{Cu}$ matrix, it is known that a spin-spiral ground state with wavevector $\vec{q}=(0.1,0,1) 2 \pi / a_{\mathrm{lat}}$ is found for $\gamma$-Fe around the lattice constant of $\mathrm{Cu}$ (Tsunoda, 1989; Tsunoda et al., 1993). Note that $q=\left(0,0,2 \pi / a_{\text {lat }}\right)$ correspond to the antiferromagnet since the distance between two layers in the $(0,0,1)$ direction of the fcc lattice is $a_{\text {lat }} / 2$. Theoretically, this wavevector was reproduced from firstprinciples calculations with good agreement where $\vec{q}=$ $(0.15,0,1) 2 \pi / a_{\text {lat }}$ (Knöpfle et al., 2000) and $\vec{q}=$ $(0.16,0,1) 2 \pi / a_{\text {lat }}$ (Sjöstedt and Nordström, 2002) were found. However, a significantly different lattice constant compared to the lattice constant of $\mathrm{Cu}$ is required for $\gamma$-Fe in the calculation compared to the experiments, which makes this agreement unsatisfactory (Sjöstedt and Nordström, 2002). In our work, instead of looking for the spin-spiral energies from firstprinciples calculations, we chose to explore the predictive power of a combination of DFT and LLG calculations for 
$\gamma$-Fe with changing lattice constants. We found a change from the low-spin to high-spin ground state in our DFT results that were performed with the JuKKR code (The JuKKR developers, 2021) through the AiiDA-KKR plugin (Rüßmann et al., 2021a; Rüßmann et al., 2021b). This agrees well with earlier DFT calculations where a similar change in the spin moment of the Fe atoms from $\mu \sim 1 \mu_{\mathrm{B}}$ to $>2.5 \mu_{\mathrm{B}}$ is seen (Knöpfle et al., 2000; Sjöstedt and Nordström, 2002).

In contrast to the spin-spiral energy calculations of Refs. (Knöpfle et al., 2000; Sjöstedt and Nordström, 2002) we calculate the exchange parameters for the extended Heisenberg model from the method of infinitesimal rotations (Liechtenstein et al., 1987) around the collinear, ferromagnetically ordered state. These parameters are then used in the SpiritCalculations where the collective magnetic ordering is investigated in a 256,000 spin supercell. We find a strong influence of exchange interactions on the lattice constant of $\gamma$-Fe which results in a competition of ferromagnetic, antiferromagnetic and spin-spiral orderings. In our analysis of the spin-spiral wavevectors we chose to study the Fourier transform of the $z$-component of the spin around the three cardinal axes which are symmetry-equivalent in our approach. At the lattice constant of $\mathrm{Cu}\left(a_{\text {lat }}=3.6 \AA\right)$ we find a spin-spiral with $\vec{q}=(0.2,0,0) 2 \pi / a_{\text {lat }}$ in contrast to the spinspiral $\vec{q}_{\text {exp }}=(0.1,0,1) 2 \pi / a_{\text {lat }}$ found experimentally which has an antiferromagnetic component (Tsunoda, 1989). We attribute this discrepancy to neglecting the change in the spin moment with the spin-spiral wavevector in our simulations based on the Heisenberg Hamiltonian. This change is known to be significant and can be as large as $0.8 \mu_{\mathrm{B}}$ (Sjöstedt and Nordström, 2002). For lattice constants $a_{\text {lat }} \leq 3.5$ we do find the correct spin-spiral with $\vec{q}=(q, 0,1) 2 \pi / a_{\text {lat }}$ that reappears after the spin-spiral with ferromagnetic ordering perpendicular to $\vec{q}$ transforms into the ordered antiferromagnet. With smaller lattice constant the spinspiral wavevector increases from $\vec{q}=(0.13,0,1) 2 \pi / a_{\text {lat }}$ at $a_{\text {lat }}=$ $3.5 \AA$ up to $\vec{q}=(0.2,0,1) 2 \pi / a_{\text {lat }}$ at $a_{\text {lat }}=3.39 \AA$ which is in reasonable agreement with earlier calculation results (Knöpfle et al., 2000; Sjöstedt and Nordström, 2002). Incorporating a change of the spin moment with the spin-spiral wavevector might further improve our agreement to the earlier $a b$ initio results of Knöpfle et al. (Knöpfle et al., 2000) and Sjöstedt and Nordström (Sjöstedt and Nordström, 2002) and also the experimental spin-spiral wavevector (Tsunoda, 1989). The need to include spin moment change is also observed in the high-pressure $\varepsilon$-phase of iron (Lebert et al., 2019). Furthermore, including higher order exchange interactions $\left(K_{i, j, k, l} \neq 0\right)$ could also be important. Especially for magnetically frustrated systems, those additional terms can be essential in describing the magnetic ground state as seen, for example, in iron chalcogenides where the biquadratic term is required for a correct description based on the Heisenberg model (Glasbrenner et al., 2015).

The change of the ordering to the antiferromagnetic state and then the reappearance of the spin-spiral state at even smaller lattice constant compared to the lattice constant of $\mathrm{Cu}$ on the other hand agrees well with the previously stated observation of competing magnetic orders, which are very close in energy and could coexist (Sjöstedt and Nordström, 2002). We further demonstrated the sensitivity of the magnetic ordering with a numerical experiment where we chose to modify the strength of the nearest neighbor exchange interaction $J_{1}$. The resulting strong change in the spin-spiral wavevector and the magnetic ordering highlights the rich energy landscape that is underlying the complex magnetic ordering in $\gamma$-Fe.

In conclusion, we have shown how augmenting spin-dynamics calculations with the Spirit code through the AiiDA-Spirit plugin enables high-throughput spin-dynamics simulations via the AiiDA infrastructure. This was applied to model systems and, in combination with DFT calculations through the AiiDA-KKR plugin, to the multi-scale problem of the magnetic ordering in $\gamma$-Fe. Our results demonstrate that typical spin-dynamics simulations benefit from the possibility to run a large number of calculations in a high-throughput fashion. Automation of SpiritCalculations through AiiDA can be a great asset when complex model parameter spaces (i.e. external fields, temperatures, different geometries, ...) are screened in order to find structure-property relations of magnetic materials. The feature of AiiDA to keep track of the data provenance is here indispensable to get reproducible results and to eventually engineer recipes for the creation and control of unconventional topological solitons in magnetic structures such as skyrmions or hopfions in the future.

\section{DATA AVAILABILITY STATEMENT}

The datasets presented in this study can be found in online repositories. The names of the repository/repositories and accession number(s) can be found below: https://archive. materialscloud.org/record/2021.203 Materials Cloud Archive 2021.203 (2021). doi: 10.24435/materialscloud:9s-tx.

\section{AUTHOR CONTRIBUTIONS}

PR and JRS programmed the first version of AiiDA-Spirit and MS and FR contributed to the further development of the plugin where FR was responsible for the spin_view functionality of AiiDA-Spirit. PR performed the DFT calculations and PR and MS performed the AiiDA-Spirit calculations. All authors discussed the results and contributed in writing the manuscript.

\section{FUNDING}

We acknowledge support by the Joint Lab Virtual Materials Design (JL-VMD) and thank for computing time granted by the JARA Vergabegremium (project number jara0191) and provided on the JARA Partition part of the supercomputer CLAIX at RWTH Aachen University. This work was funded by the Deutsche Forschungsgemeinschaft (DFG, German Research Foundation) under Germany's Excellence Strategy-Cluster of Excellence Matter and Light for Quantum Computing (ML4Q) EXC 2004/1-390534769. 


\section{REFERENCES}

Back, C., Cros, V., Ebert, H., Everschor-Sitte, K., Fert, A., Garst, M., et al. (2020). The 2020 Skyrmionics Roadmap. J. Phys. D: Appl. Phys. 53, 363001. doi:10. 1088/1361-6463/ab8418

Bessarab, P. F., Uzdin, V. M., and Jónsson, H. (2012). Harmonic Transition-State Theory of thermal Spin Transitions. Phys. Rev. B 85, 184409. doi:10.1103/ physrevb.85.184409

Bessarab, P. F., Uzdin, V. M., and Jónsson, H. (2015). Method for Finding Mechanism and Activation Energy of Magnetic Transitions, Applied to Skyrmion and Antivortex Annihilation. Comput. Phys. Commun. 196, 335-347. doi:10.1016/j.cpc.2015.07.001

Binder, K., and Heermann, D. W. (1997). Monte Carlo Simulation in Statistical Physics. Berlin: Springer-Verlag.

Bogolubsky, I. L. (1988). Three-dimensional Topological Solitons in the Lattice Model of a Magnet with Competing Interactions. Phys. Lett. A 126, 511-514. doi:10.1016/0375-9601(88)90049-7

Depondt, P., and Mertens, F. G. (2009). Spin Dynamics Simulations of TwoDimensional Clusters with Heisenberg and Dipole-Dipole Interactions. J. Phys. Condens. Matter 21, 336005. doi:10.1088/0953-8984/21/33/336005

Dupé, B., Hoffmann, M., Paillard, C., and Heinze, S. (2014). Tailoring Magnetic Skyrmions in Ultra-thin Transition Metal Films. Nat. Commun. 5, 4030-4036. doi: $10.1038 /$ ncomms 5030

Ebert, H., Ködderitzsch, D., and Minár, J. (2011). Calculating Condensed Matter Properties Using the KKR-Green's Function Method-Recent Developments and Applications. Rep. Prog. Phys. 74, 096501. doi:10.1088/0034-4885/74/9/ 096501

Gilbert, T. L. (2004). Classics in Magnetics A Phenomenological Theory of Damping in Ferromagnetic Materials. IEEE Trans. Magn. 40, 3443-3449. doi:10.1109/tmag.2004.836740

Glasbrenner, J. K., Mazin, I. I., Jeschke, H. O., Hirschfeld, P. J., Fernandes, R. M., and Valentí, R. (2015). Effect of Magnetic Frustration on Nematicity and Superconductivity in Iron Chalcogenides. Nat. Phys 11, 953-958. doi:10.1038/ nphys3434

Heinze, S., Von Bergmann, K., Menzel, M., Brede, J., Kubetzka, A., Wiesendanger, R., et al. (2011). Spontaneous Atomic-Scale Magnetic Skyrmion Lattice in Two Dimensions. Nat. Phys 7, 713-718. doi:10.1038/nphys 2045

Himanen, L., Geurts, A., Foster, A. S., and Rinke, P. (2019). Data-Driven Materials Science: Status, Challenges, and Perspectives. Adv. Sci. 6, 1900808. doi:10.1002/ advs. 201900808

Hoffmann, M., and Blügel, S. (2020). Systematic Derivation of Realistic Spin Models for Beyond-Heisenberg Solids. Phys. Rev. B 101, 024418. doi:10.1103/ physrevb.101.024418

Hoffmann, M., Zimmermann, B., Müller, G. P., Schürhoff, D., Kiselev, N. S., Melcher, C., et al. (2017). Antiskyrmions Stabilized at Interfaces by Anisotropic Dzyaloshinskii-Moriya Interactions. Nat. Commun. 8, 308-309. doi:10.1038/ s41467-017-00313-0

Hoffmann, M., Müller, G. P., Melcher, C., and Blügel, S. (2021). Skyrmionantiskyrmion Racetrack Memory in Rank-One Dmi Materials. Front. Phys. 9, 668. doi:10.3389/fphy.2021.769873

Huber, S. P., Zoupanos, S., Uhrin, M., Talirz, L., Kahle, L., Häuselmann, R., et al. (2020). AiiDA 1.0, a Scalable Computational Infrastructure for Automated Reproducible Workflows and Data Provenance. Sci. Data 7, 300. doi:10.1038/ s41597-020-00638-4

Kent, N., Reynolds, N., Raftrey, D., Campbell, I. T. G., Virasawmy, S., Dhuey, S., et al. (2021). Creation and Observation of Hopfions in Magnetic Multilayer Systems. Nat. Commun. 12, 1562-1567. doi:10.1038/s41467021-21846-5

Knöpfle, K., Sandratskii, L. M., and Kübler, J. (2000). Spin Spiral Ground State of $\gamma$-Iron. Phys. Rev. B 62, 5564-5569. doi:10.1103/PhysRevB.62.5564

Krönlein, A., Schmitt, M., Hoffmann, M., Kemmer, J., Seubert, N., Vogt, M., et al. (2018). Magnetic Ground State Stabilized by Three-Site Interactions: Fe/Rh(111). Phys. Rev. Lett. 120, 207202. doi:10.1103/physrevlett.120. 207202

Landau, L., and Lifshitz, E. (1935). On the Theory of the Dispersion of Magnetic Permeability in Ferromagnetic Bodies. Phys. Z. Sowjet. 851, 153.
Lebert, B. W., Gorni, T., Casula, M., Klotz, S., Baudelet, F., Ablett, J. M., et al (2019). Epsilon Iron as a Spin-Smectic State. Proc. Natl. Acad. Sci. USA 116, 20280-20285. doi:10.1073/pnas.1904575116

Liechtenstein, A. I., Katsnelson, M. I., Antropov, V. P., and Gubanov, V. A. (1987). Local Spin Density Functional Approach to the Theory of Exchange Interactions in Ferromagnetic Metals and Alloys. J. Magnetism Magn. Mater. 67, 65-74. doi:10.1016/0304-8853(87)90721-9

Müller, G. P., Sallermann, M., Mavros, S., Rhiem, F., Schürhoff, D., Meyer, I., et al. (2021). Spirit: Spin Simulation Software. Available at: https://github.com/spiritcode/spirit (Accessed November 25, 2021).

Müller, G. P., Hoffmann, M., Dißelkamp, C., Schürhoff, D., Mavros, S., Sallermann, M., et al. (2019). Spirit : Multifunctional Framework for Atomistic Spin Simulations. Phys. Rev. B 99, 224414. doi:10.1103/ PhysRevB.99.224414

Muhlbauer, S., Binz, B., Jonietz, F., Pfleiderer, C., Rosch, A., Neubauer, A., et al. (2009). Skyrmion Lattice in a Chiral Magnet. Science 323, 915-919. doi:10.1126/ science. 1166767

Perdew, J. P., Burke, K., and Ernzerhof, M. (1996). Generalized Gradient Approximation Made Simple. Phys. Rev. Lett. 77, 3865-3868. doi:10.1103/ PhysRevLett.77.3865

Pizzi, G., Cepellotti, A., Sabatini, R., Marzari, N., and Kozinsky, B. (2016). AiiDA: Automated Interactive Infrastructure and Database for Computational Science. Comput. Mater. Sci. 111, 218-230. doi:10.1016/j. commatsci.2015.09.013

Rüßmann, P., Bertoldo, F., Bröder, J., Wasmer, J., Mozumder, R., Chico, J., et al. (2021). JuDFTteam/aiida-kkr: AiiDA Plugin for the JuKKR Codes. doi:10.5281/ zenodo.3628251

Rüßmann, P., Bertoldo, F., and Blügel, S. (2021). The AiiDA-KKR Plugin and its Application to High-Throughput Impurity Embedding into a Topological Insulator. Npj Comput. Mater. 7, 13. doi:10.1038/s41524020-00482-5

Rüßmann, P., Ribas Sobreviela, J., Sallermann, M., Hoffmann, M., Rhiem, F., and Blügel, S. (2021b). The AiiDA-Spirit Plugin for Automated SpinDynamics Simulations and Multi-Scale Modelling Based on FirstPrinciples Calculations. Mater. Cloud Archive, 203. doi:10.24435/ materialscloud:9s-tx

Sjöstedt, E., and Nordström, L. (2002). Noncollinear Full-Potential Studies of $\gamma-F e$ Phys. Rev. B 66, 014447. doi:10.1103/PhysRevB.66.014447

Skubic, B., Hellsvik, J., Nordström, L., and Eriksson, O. (2008). A Method for Atomistic Spin Dynamics Simulations: Implementation and Examples. J. Phys. Condens. Matter 20, 315203. doi:10.1088/0953-8984/20/31/ 315203

Stefanou, N., Akai, H., and Zeller, R. (1990). An Efficient Numerical Method to Calculate Shape Truncation Functions for Wigner-Seitz Atomic Polyhedra. Comput. Phys. Commun. 60, 231-238. doi:10.1016/00104655(90)90009-P

Stefanou, N., and Zeller, R. (1991). Calculation of Shape-Truncation Functions for Voronoi Polyhedra. J. Phys. Condens. Matter 3, 7599-7606. doi:10.1088/0953$8984 / 3 / 39 / 006$

Sutcliffe, P. (2018). Hopfions in Chiral Magnets. J. Phys. A: Math. Theor. 51, 375401. doi:10.1088/1751-8121/aad521

Szilva, A., Costa, M., Bergman, A., Szunyogh, L., Nordström, L., and Eriksson, O. (2013). Interatomic Exchange Interactions for Finite-Temperature Magnetism and Nonequilibrium Spin Dynamics. Phys. Rev. Lett. 111, 127204. doi:10.1103/ physrevlett.111.127204

Talirz, L., Kumbhar, S., Passaro, E., Yakutovich, A. V., Granata, V., Gargiulo, F., et al. (2020). Materials Cloud, a Platform for Open Computational Science. Sci. Data 7, 299. doi:10.1038/s41597-020-00637-5

The AiiDA-Spirit developers (2021). The Aiida-Spirit Plugin. Available at: https:// github.com/JuDFTteam/aiida-spirit (Accessed November 25, 2021)

The AiiDA team (2021). AiiDA Plugin Registry. Available at: https://aiidateam. github.io/aiida-registry/ (Accessed November 25, 2021).

The JuKKR developers (2021). The Jülich KKR Codes. Available at: https://jukkr. fz-juelich.de (Accessed November 25, 2021).

Tsunoda, Y., Nishioka, Y., and Nicklow, R. M. (1993). Spin Fluctuations in Small $\gamma$-Fe Precipitates. J. Magnetism Magn. Mater. 128, 133-137. doi:10.1016/ 0304-8853(93)90867-2 
Tsunoda, Y. (1989). Spin-density Wave in Cubic $\gamma$-Fe and $\gamma \mathrm{Fe}_{100-\mathrm{x}} \mathrm{Co}_{\mathrm{x}}$ precipitates in Cu. J. Phys. Condens. Matter 1, 10427-10438. doi:10.1088/0953-8984/1/ $51 / 015$

Uhrin, M., Huber, S. P., Yu, J., Marzari, N., and Pizzi, G. (2021). Workflows in AiiDA: Engineering a High-Throughput, Event-Based Engine for Robust and Modular Computational Workflows. Comput. Mater. Sci. 187, 110086. doi:10. 1016/j.commatsci.2020.110086

Vfrendering (2021). A Vector Field Rendering Library. Available at: https://github. com/FlorianRhiem/VFRendering (Accessed November 25, 2021).

Weißenhofer, M., Rózsa, L., and Nowak, U. (2021). Skyrmion Dynamics at Finite Temperatures: Beyond Thiele's Equation. Phys. Rev. Lett. 127, 047203. doi:10. 1103/PhysRevLett.127.047203

Wilkinson, M. D., Dumontier, M., Aalbersberg, I. J., Appleton, G., Axton, M., Baak, A., et al. (2016). The FAIR Guiding Principles for Scientific Data Management and Stewardship. Sci. Data 3, 160018. doi:10.1038/sdata. 2016.18

Xu, Q., Bergman, A., Delin, A., and Chico, J. (2021). The UppASD-AiiDA Plugin. Available at: https://github.com/UppASD/aiida-uppasd (Accessed November 25, 2021).
Yu, X. Z., Onose, Y., Kanazawa, N., Park, J. H., Han, J. H., Matsui, Y., et al. (2010). Real-space Observation of a Two-Dimensional Skyrmion crystal. Nature 465, 901-904. doi:10.1038/nature09124

Conflict of Interest: The authors declare that the research was conducted in the absence of any commercial or financial relationships that could be construed as a potential conflict of interest.

Publisher's Note: All claims expressed in this article are solely those of the authors and do not necessarily represent those of their affiliated organizations, or those of the publisher, the editors, and the reviewers. Any product that may be evaluated in this article, or claim that may be made by its manufacturer, is not guaranteed or endorsed by the publisher.

Copyright (C) 2022 Rüßmann, Ribas Sobreviela, Sallermann, Hoffmann, Rhiem and Blügel. This is an open-access article distributed under the terms of the Creative Commons Attribution License (CC BY). The use, distribution or reproduction in other forums is permitted, provided the original author(s) and the copyright owner(s) are credited and that the original publication in this journal is cited, in accordance with accepted academic practice. No use, distribution or reproduction is permitted which does not comply with these terms. 